\title{
Acoustic Metamaterials in Aeronautics
}

\author{
Giorgio Palma 1,*(D), Huina Mao ${ }^{2}$, Lorenzo Burghignoli ${ }^{1}$, Peter Göransson 2 (1) \\ and Umberto Iemma ${ }^{1}$ (i) \\ 1 Department of Engineering, Roma Tre University, 00146 Roma, Italy; lorenzo.burghignoli@uniroma3.it (L.B.); \\ umberto.iemma@uniroma3.it (U.I.) \\ 2 Department of Aeronautical and Vehicle Engineering, KTH Royal Institute of Technology, \\ Stockholm SE-100 44, Sweden; huina@kth.se (H.M.); pege@kth.se (P.G.) \\ * Correspondence: giorgio.palma@uniroma3.it; Tel.:+39 0657333302
}

Received: 4 May 2018; Accepted: 7 June 2018; Published: 13 June 2018

\begin{abstract}
Metamaterials, man-made composites that are scaled smaller than the wavelength, have demonstrated a huge potential for application in acoustics, allowing the production of sub-wavelength acoustic absorbers, acoustic invisibility, perfect acoustic mirrors and acoustic lenses for hyper focusing, and acoustic illusions and enabling new degrees of freedom in the control of the acoustic field. The zero, or even negative, refractive sound index of metamaterials offers possibilities for the control of acoustic patterns and sound at sub-wavelength scales. Despite the tremendous growth in research on acoustic metamaterials during the last decade, the potential of metamaterial-based technologies in aeronautics has still not been fully explored, and its utilization is still in its infancy. Thus, the principal concepts mentioned above could very well provide a means to develop devices that allow the mitigation of the impact of civil aviation noise on the community. This paper gives a review of the most relevant works on acoustic metamaterials, analyzing them for their potential applicability in aeronautics, and, in this process, identifying possible implementation areas and interesting metabehaviors. It also identifies some technical challenges and possible future directions for research with the goal of unveiling the potential of metamaterials technology in aeronautics.
\end{abstract}

Keywords: metamaterials; aviation noise; aeroacoustics; noise absorption; noise reflection; noise trapping; acoustic cloaking

\section{Introduction}

The first definitions for the term metamaterial were given by researchers in the electromagnetic (EM) field. In 1999, Wieglhofer and Lakhtakia [1] defined a metamaterial as a "three-dimensional, periodic cellular architecture, designed to produce an optimized combination not available in nature of two or more responses to a specific excitation". This definition was completed and extended by Cui, Liu and Smith [2] in 2010 who stated that a metamaterial is "a macroscopic composite of periodic or non-periodic structures whose function is due to both its cellular architecture and chemical composition". From these principal definitions, there emerges the possibility to achieve properties that are hard or impossible to find in nature by conventionally engineered materials. Metamaterials derive their properties from their designed structures and geometries more than from their chemical composition, and the response of properly defined unit cells can be translated into averaged effective parameters, e.g., an effective density and effective bulk modulus.

Research on metamaterials started in the electromagnetic field. Veselago [3] first demonstrated analytically that a negative refraction index is attainable if a medium exhibits both negative permeability and negative permittivity simultaneously, and this preliminary concept was experimentally realized by Pendry [4] in 2000, when a superlens was proposed, exhibiting a negative refraction index in the frequency bandwidth of visible light or microwaves [4]. 
The results of this left handed behavior of negatively refracting metamaterials obtained in the EM field sparked the interest of the scientific community in other fields, such as (but not only) acoustics. This was the case for the early work by Liu et al. [5], who proposed an acoustic metamaterial that attenuates noise in the mid-frequency range (40 to $1100 \mathrm{~Hz}$ ), which is composed of periodically-arranged, high density, spherical cores (made of lead), uniformly coated with a layer of soft and elastic material (silicone rubber). In 2004, Li and Chan [6] mathematically demonstrated, for the first time, the possibility of designing a metamaterial to achieve a negative effective bulk modulus and density, acoustically introducing slow inclusions in a faster medium. In a frequency range such that the wavelength inside one inclusion is comparable to the dimension of the inclusion itself, but still much larger than the distance between the inclusions, the effective density and bulk modulus of the metamaterial appear to be negative $[7,8]$.

Physically, a double negative metamaterial shows counterintuitive behaviors-it expands when compressed and reacts with an acceleration in phase opposition with respect to the force that induces it. This consideration better explains the meaning of negative density, which reads asthe negative dynamic mass density term in Newton's second law $[9,10]$.

When both mass density $(\rho)$ and the bulk modulus $(\mathcal{K})$ are negative, an acoustic wave is allowed to propagate inside the medium, thus exhibiting a negative refractive index. This causes energy to flow in the opposite direction with respect to the wave, i.e., the wave vector and the Poynting vector (describing the energy flow associated with the acoustic propagation) point in opposite directions. A metamaterial exposing the negative refraction index can be exploited for applications like acoustic superlenses [4,11], i.e., a device capable of focusing an incident acoustic wave (with a sub-wavelength spacial resolution), even beyond the diffraction limit, in a sub-wavelength spot, and, in general, for bending waves and shaping the acoustic field almost arbitrarily.

Near-total reflection can be obtained when only one of the two material parameters, $\rho$ and $\mathcal{K}$, becomes negative. This can be achieved using resonant elements to let $\rho$ turn negative [5], or with a membrane decorated with a resonant mass(es) [12-15], even without [16,17]. The frequency working range can be tuned, adjusting the weight of the masses, if present, or other constructive parameters to place the resonant effect even in the low frequency range. Stacking more than one metamaterial panel together can be a strategy to obtain a more broadband effect.

Another type of singular negative metamaterial can be obtained when the effective bulk modulus turns negative. In Fang et al. [18], inspired by results obtained in the electromagnetic field, an array of sub-wavelength Helmholtz resonators is used to obtain this effect in the ultrasonic regime. Dipolar and monopolar local resonances were proved to be the essential wave mechanisms for producing, respectively, a negative effective mass density and a negative effective bulk modulus of the metamaterial.

Double negative acoustic metamaterials can be achieved by combining negative density and negative compressibility materials, or by overlapping monopolar and dipolar resonances in the same frequency range, or to design phononic crystals to generate a band folding through multiple scattering in a periodic structure.

Different and more complex devices with equivalent dynamic or electromagnetic lumped models have been developed so far [19] to obtain metamaterials, based on locally resonant elements that show single or double negativity, depending on the considered frequency working range [19-21].

Other approaches have also been successfully adopted to realize metamaterials with interesting properties, such as coiling up space by using labyrinthine structures to introduce local phase delay in sound propagation. The phase shift can be designed in the full $0-2 \pi$ range to obtain flat surfaces to act as arbitrarily-shaped virtual surfaces [22-24], modifying the reflection angle, or it could be random to maximize acoustic diffusion $[25,26]$ (Figure 1). Space-coiling metamaterials can be adopted to obtain lens-like behaviors or even to transform the propagation pattern from spherical to plane waves $[27,28]$. Furthermore, this approach allows for an effective dynamic $\rho<0$ and $\mathcal{K}<0$, and hence, a negative refractive index to be obtained in the deep sub-wavelength regime with a quite simple device $[29,30]$. 
When a metamaterial device is built with a thickness below a tenth of the working wavelength, it is commonly called a metasurface. There is obviously a great interest in developing metasurfaces, as a reduced thickness is often desirable for real applications. Coiled-up space devices and resonant membranes were intrinsically designed to lead to metasurfaces, and hence, the majority of the so-based work presented in previous paragraphs classifies as metasurfaces, due to their deep sub-wavelength thickness. However, for each of the approaches presented above, some engineered metasurfaces can be found in the literature [31-35].

\section{Normal design}

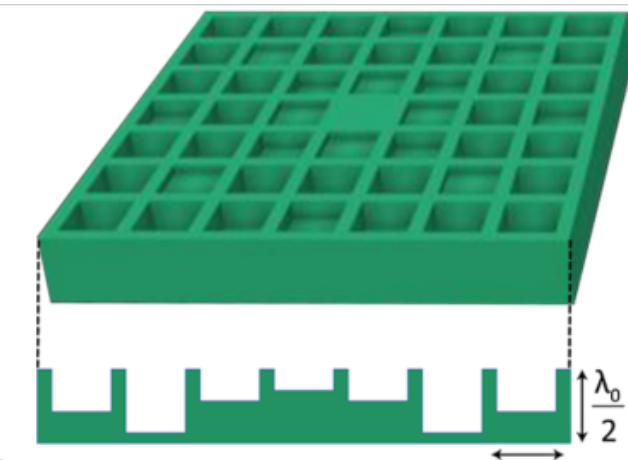

a)

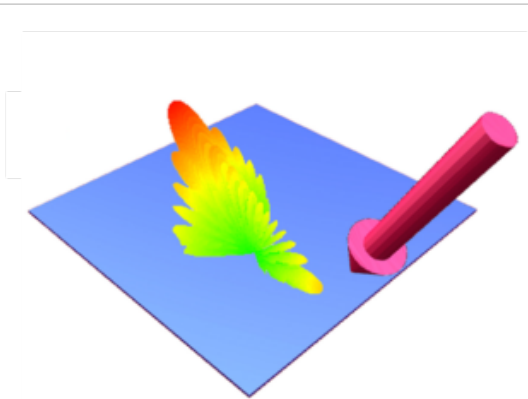

Flat plate

\section{b)}

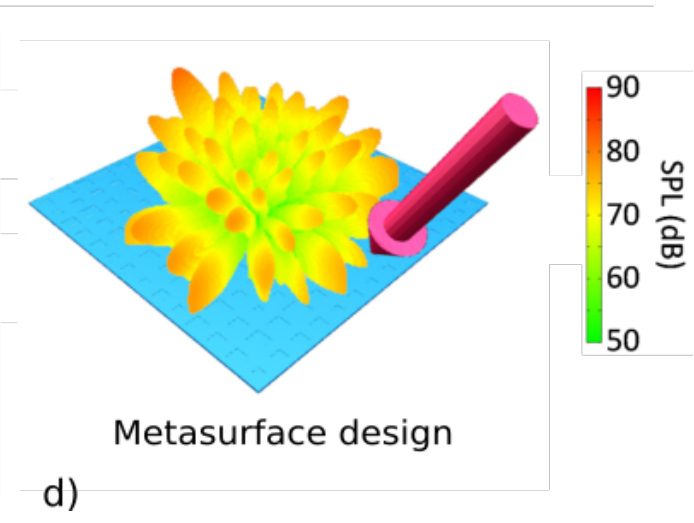

Metasurface design

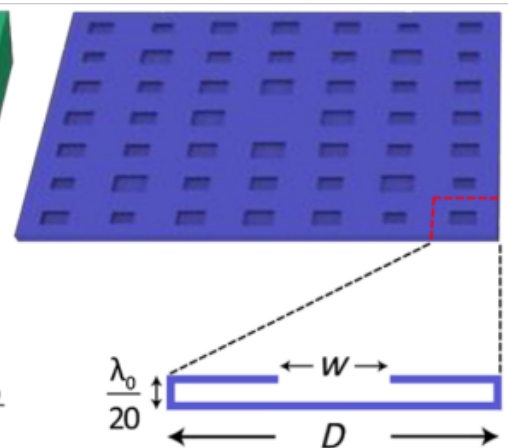

b)

c)

Figure 1. Metasurface design of a Schroeder Diffuser from [26]: diffuse scattering is achieved through a deep sub-wavelength metasurface. Each cell adds a different and random arranged phase-shift in the reflected field. In $(\mathbf{a}, \mathbf{b})$ the standard and the metasurface designs are compared. The simulated far-field scattering pattern for a flat plate and for the metasurface diffuser are shown in (c,d) in terms of sound pressure level (the arrow indicates the incident acoustic perturbation) evidencing the achieved metabehavior.

A very interesting superabsorber with micro-bubble insertions was presented in [36]. A significant sub-wavelength attenuation of sound was shown due to the resonance of the bubbles in a soft elastic matrix, breaking the mass density law (which states that the amplitude of transmittance for a solid panel is proportional to the inverse of the product of the density and the thickness of the panel and the frequency of the acoustic perturbation).

Thermo-viscous losses at the solid-fluid interfaces inside metamaterial components are crucial in determining the behavior of metamaterials. This is particularly true for concepts that consider micro-slits, micro-cavities and/or narrow channels as part of the design, like space-coiling metamaterials. The visco-thermal loss mechanism becomes non-negligible even when the widths of the slits are about two order of magnitude bigger than the viscous and thermal boundary layers (in the order of $10^{-5} \mathrm{~m}$ ) and is enhanced by the fact that high amplitude standing waves can form in small cavities. Such losses can deeply modify the expected behavior of the metamaterial under study and hence, should be carefully taken into account and exploited when dissipation and absorption 
are sought $[37,38]$. The geometric parameters of a metamaterial can even be tuned to achieve high transmission, high absorption or high reflection from the same basic concept [39].

The great potential of acoustic metamaterials has not yet been totally disclosed, and this is even more true when thinking about aeroacoustic and aeronautical applications. This paper will highlight interesting behaviors and promising concepts, focusing on the reduction of the community noise produced by aircrafts, and will also show some interesting cabin noise reduction applications. Section 2 considers different metabehaviors, introducing their possible applications for aircrafts and the sought effect, specifically Section 2.1 focuses on absorption, Section 2.2 focuses on unusual reflection by metasurfaces, Section 2.3 focuses on noise trapping and Section 2.4 focuses on scattering abatement from objects, i.e., acoustic cloaking. For each subsection, the most promising existing concept is shown, emphasizing possible limitations and/or potential improvements needed for its implementation on aircrafts. Section 3 addresses challenges that need to be addressed in the near future by researchers in order to make metamaterial technologies mature enough for aeronautical industrial applications, so that they achieve a higher Technology Readiness Level (TRL), and finally some conclusion remarks can be found in Section 4 .

\section{Potential Applications of Aeroacoustic Metamaterials}

Sustainable development of the air transport system implies that the fundamental issues related to the rapid and continuous expansion of the urban areas surrounding airports, and, in parallel, the increase in air traffic increasing the community noise levels, both have to be tackled. This has been the driving force over the last decade that has led national and international funding bodies throughout the world to provide increasing financial support to research projects focused on the mitigation of the impact of aeronautical noise on the residential community (see, e.g., the call for Mobility for Growth within the context of the Small, Green and Integrated Transport challenge of the Horizon 2020 Program). Airlines also have an economic interest in operating quieter aircraft, as airport authorities impose the payment of fees proportional to the noise generated by their airplanes and in the future, property taxes will be introduced for owners of noisy aircraft to encourage the innovation of operating fleets and the spreading of the most advanced noise-reduction technologies. This development scenario is worsened by the apparent stagnation in the development of new technologies typically adopted on commercial aircraft, thus imposing the need for a breakthrough to guarantee further reductions in noise emissions. Metamaterials may very well prove to be the breakthrough technology needed to advance beyond the current state-of-the-art in noise reduction technology.

The introduction of any type of metamaterial-based device in aeronautical applications is restricted in several different ways. The first condition that has to be met is the weight: an excessive weight penalty is, in general, not acceptable as it would compromise the performance and fuel efficiency of the aircraft. For this reason, concepts involving a high mass density will be excluded from our analysis. As far as community noise is concerned, take-off and landing are the most critical phases of an aircraft operational cycle. Most of the noise emitted comes from the propulsion system and the high lift devices required during climb and descent. From these particular source locations, the application of metamaterials aimed at providing significant noise reductions could be the engine nacelle, the internal ducts of the engine itself, and the trailing and side edges of the wings and flaps.

\subsection{Absorption and Dissipation}

Typically, the strategy adopted to reduce the exposure of citizens to aircraft noise is based on the minimization of the sound energy at ground location, hence shrinking the area inside the critical isophonic curves. Reducing the noise level provides a major contribution to the alleviation of community noise [40].

As stated above, engines are a significant source of noise under all flight conditions, largely dominating during take-off and being comparable to airframe noise during landing. Among the engine sources, fan noise is one of the biggest component in the overall noise (see Figure 2), and its 
relative importance has increased in the last twenty years as the bypass ratio of modern turbofan engines have increased. This latter development has been led by the need to reduce the specific fuel consumption, but has, as a side effect, also reduced the jet noise, in comparison to the other noise components.

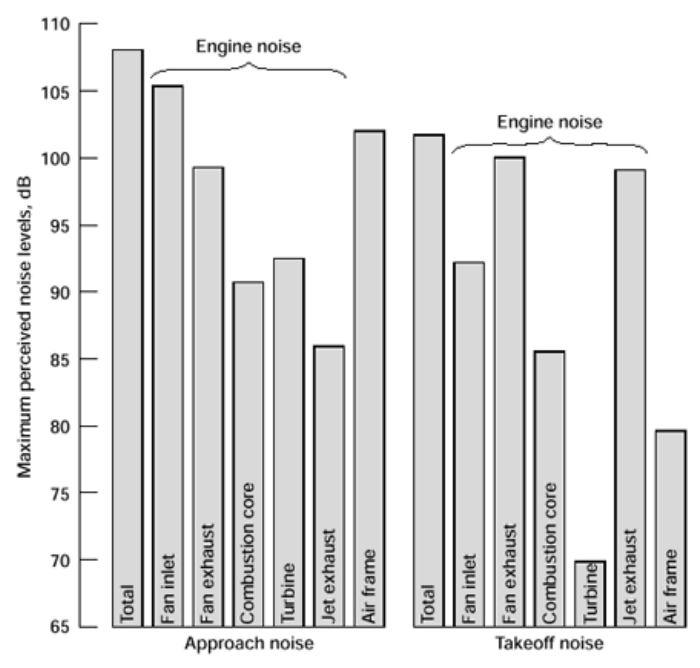

Figure 2. Typical distribution of aircraft noise between different sources during take-off and approach. Picture from [41].

For modern aircraft, most of the engine noise attenuation is provided by liners, which are absorbing panels positioned in the internal walls of the nacelle that are designed to reduce both broadband and tonal noise. The basic single degree-of-freedom liner is typically made by an hard-backed honeycomb and behaves like a quarter-wavelength resonator, covered by a perforated plate that, on one side, enhances the sound absorption of the liner and on the other, maintains the aerodynamic flow as cleanly as possible on the walls of the nacelle. Liner design is one of the most active research field in aeronautics and recently, metamaterials have started to be taken into consideration for future and more effective absorbing panels for liners, adopting e.g., dual-resonant materials covered by microperforated panels $[42,43]$ or variable depth liners with spherical inclusions and microporous panels embedded within melamine [44].

The most desired effect that could be possibly achieved by adopting metamaterial-inspired liners is low frequency absorption, i.e., in the range of $100-1000 \mathrm{~Hz}$, which is difficult to achieve with classical approaches, due to the long wavelengths of sound involved at those frequencies. Several authors have proposed new metamaterials for low frequency noise absorption that are of interest and could possibly be applied to the design of new liners. Some example sare shown in Figure 3, such as thin film membranes with rigid platelets, as presented in [15], in which the incident acoustic wave is dissipated at the resonance of a cavity system due to enhanced energy concentration at discontinuities in the slope of the displacement profile. Space-coiling metamaterials have been proposed as dissipative media with proven sound absorption ability, in combination with microperforated panel coverings $[39,45,46]$. One of the advantages promised by such design is a deep sub-wavelength functionality, often reached by exploiting Fabry-Pérot resonance in long, coiled, narrow channels (Figure 4), which enhance dissipation phenomena at resonant frequency. Another valuable contribution in this field is the work by Yang et al. [47], who derived a "causality" constraint that dictates trade-off conditions between the sample thickness, frequency bandwidth and magnitude of absorption in the low frequency range and presented a design strategy to realize the target absorption coefficient profile with the minimum possible thickness.

Resonance and viscous dissipation was also exploited in a very interesting design by Tang et al. [48]. A series of different acoustic Helmholtz resonators was obtained by inserting inclined 
microporous septa in a honeycomb structure with a microperforated panel on top (see Figure 5). The corrugated structure exhibited several resonant frequencies that caused the air in the perforation to oscillate severely when excited, thus dissipating energy in the viscous boundary layer, obtaining a broadband high absorption coefficient for a wide range of incident angles of acoustic perturbation.

a)
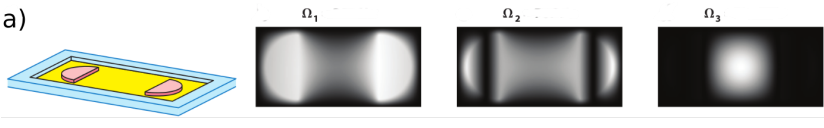

b)
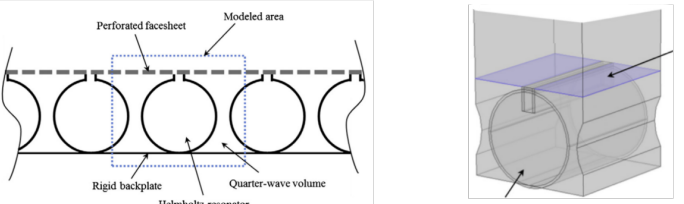

c)

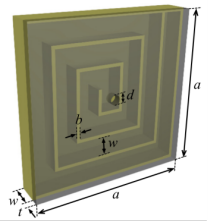

d)

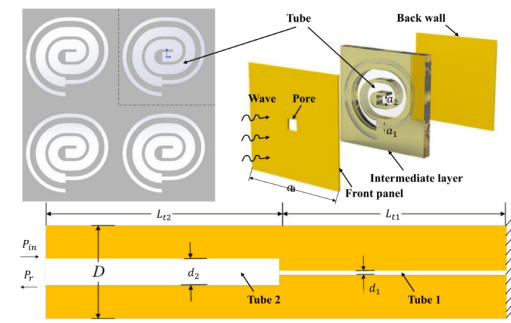

Figure 3. Various metamaterial designs for low-frequency absorption: (a) a membrane decorated with added platelets and displacement related to the first three eigenfrequencies of the structure from [47]; (b) a dual resonant metamaterial composed of Helmholtz's resonators, and a perforated sheet adopted as an innovative liner in [43]; (c,d) two different space-coiling designs, respectively from [45] and [46]. The dissipation of acoustic energy is fundamental in each design.

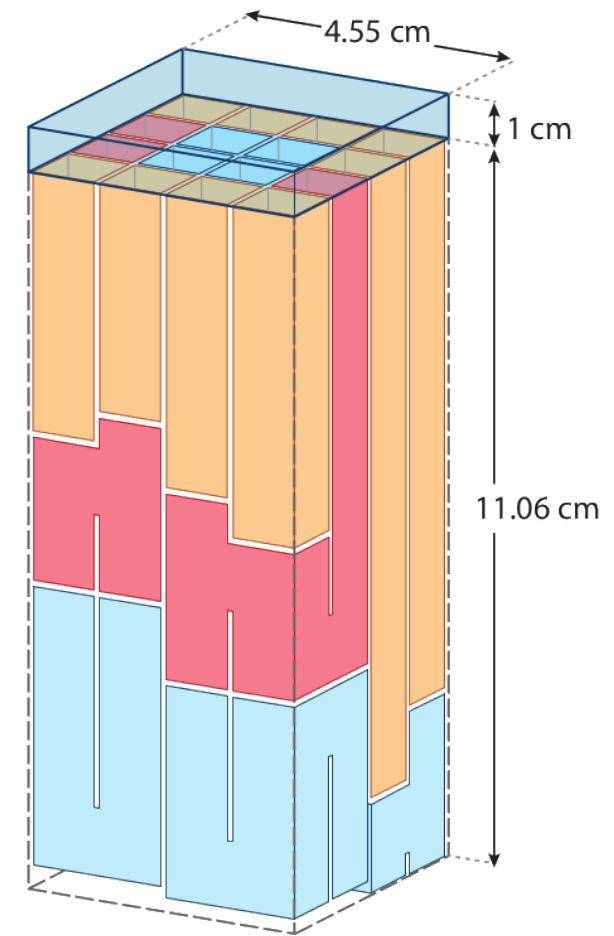

Figure 4. The original "optimal" absorbing metamaterial obtained by Yang et al. The unit cell is composed of 16 resonant channels differing in length and folded to compact the sample (image from [47]). 


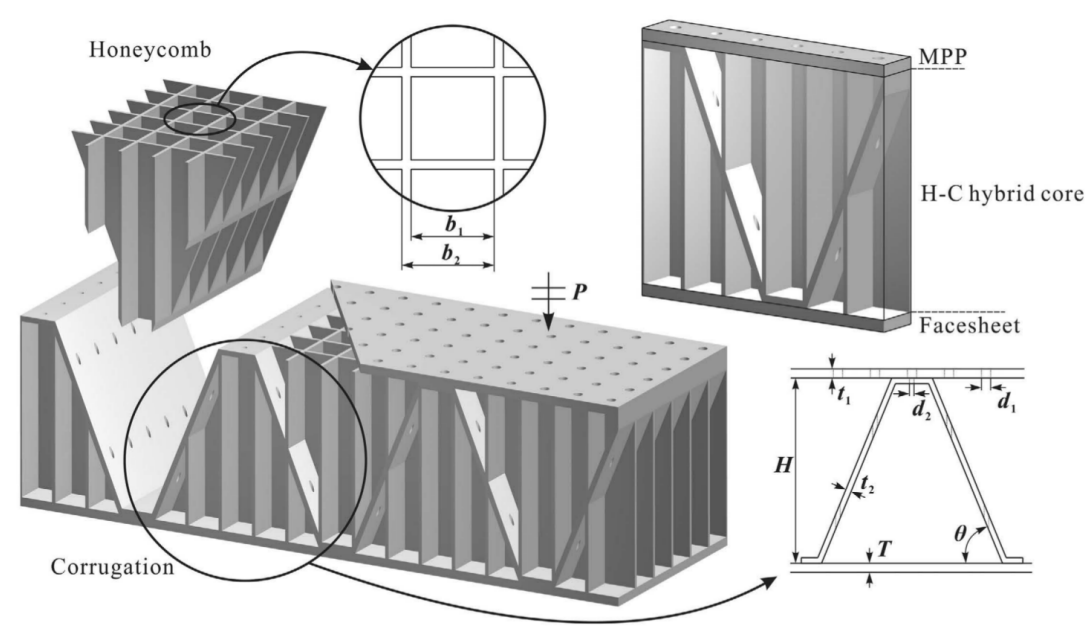

Figure 5. Image from [48]. An absorbing metamaterial composed of various different Helmholtz resonators separated by microporous septa. The reference values for parameters are $d_{1}, t_{2}, d_{2}, T=1 \mathrm{~mm}$, $t_{1}=2 \mathrm{~mm}, b_{1}=3.6 \mathrm{~mm}, b_{2}=4 \mathrm{~mm}, H=20 \mathrm{~mm}$, and these can be tuned to obtain the maximum absorption at the desired frequency (e.g., an absorption peak at $580 \mathrm{~Hz}$ is obtained with $\mathrm{H}=60 \mathrm{~mm}$ $d_{2}=0.2 \mathrm{~mm}$ and $d_{1}=1.3 \mathrm{~mm}$ ).

The annoyance from a noise, however, is not only related to its intensity. The mathematical description of an annoyance is not a simple task, due to the subjectivity of the sound event perception, which is affected by non-acoustical factors that have not yet fully been enlightened and are still an object of study. Projects like SEFA (Sound Engineering for Aircraft) and COSMA (Community Oriented Solutions to Minimise aircraft noise Annoyance) have demonstrated that the spectral content of the signal is fundamental in defining its effect on listeners. Extensive psychometric test campaigns performed during the projects were able to identify weakly-annoying target sounds that were usually characterized by attenuated tonal components drawn in broadband noise. This illustrates how important it is to enhance the acoustic absorption at specific frequencies to improve the quality of sound/noise from an aircraft and hence, its acceptability by the community, i.e., reducing acoustic pollution, even while maintaining the sound level. Such devices would enable the possibility of engineering the noise signature from an aircraft and hence, rethinking the conceptual design procedure by including the "desired noise" as a variable to optimize [49-51].

\subsection{Reflection}

A smart way to reduce the incidence of noise pollution from an aircraft is to deflect the sound waves in order to redirect them away from the ground and community (a qualitative directivity pattern of noise emitted by turbofan components can be found in Figure 6). Upward redirection of part of the emitted acoustic energy has been tested in the past using negatively scarfed nacelle inlets. The main characteristic of such engine intakes is to have the bottom part longer than the upper, causing greater reflection of acoustic waves to the upper direction. Despite the promising community noise reduction achievable, aerodynamic side effects of such designs have been found to be too severe, affecting the overall performance of the aircraft, and thus making its usage unsuitable [52]. Also, here, metamaterials that would be able to effectively modify the reflection of acoustic waves could be candidates for engineered "virtually scarfed nacelles", obtaining the desired acoustic effect without compromising the inlet shape, which is still be optimized for aerodynamic and engine efficiency. 


\section{Turbofan Noise directivity}

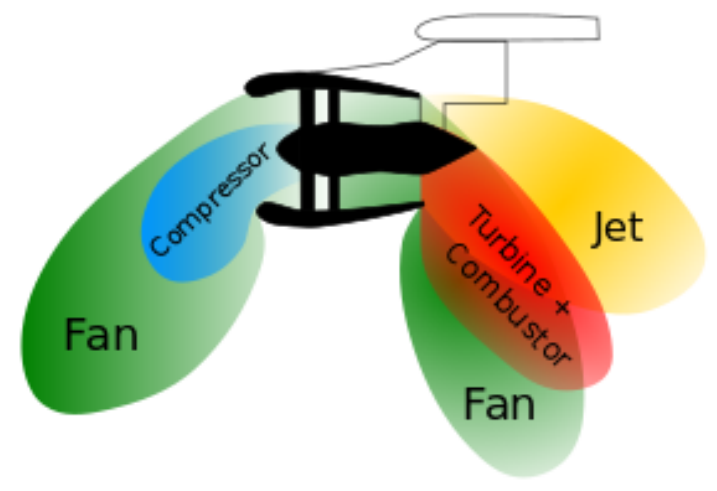

Figure 6. Typical noise directivity from components of a modern turbofan.

Many of the space-coiling metamaterials studied in the papers mentioned in the introduction have shown intriguing and abnormal reflection capabilities. In the authors' opinion, one of the most interesting contribution was presented in the works by $\mathrm{Li}$ et al. both numerically and experimentally [22,23]. The main idea was to impose a phase shift profile to the reflected field by properly selecting geometrical parameters of an ultrathin, planar metasurface, whose elementary unit was made of two stiff "corrugated beams" with a hard back-end (Figure 7). A reduced wall thickness and overall depth (in the order of $1 / 20$ of wavelength) are strengths of the proposed approach, together with the ease of tuning of the design to work at a desired frequency. Clearly, a continuous phase profile is not achievable with the proposed design due to the necessary width of the unit cell. The cited works $[22,23]$ successfully adopted a $\pi / 4$ discretization step over the $2 \pi$ range. The ideal theoretical phase profile was hence approximated using eight unit cells to provide discrete phase shifts. When a sound wave enters a cell, it is forced to follow a longer path with respect to the thickness of the cell itself, coming out with a phase delay directly connected to the equivalent straight path of the considered cell.

a)
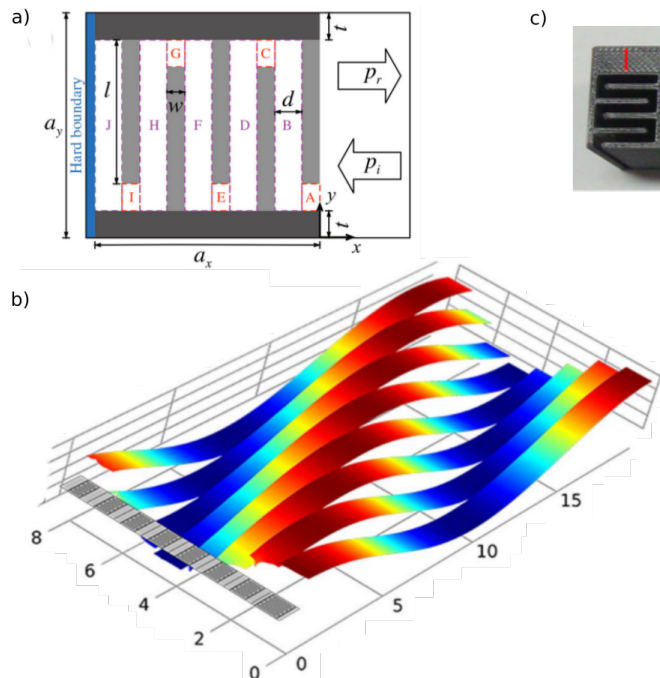

c)

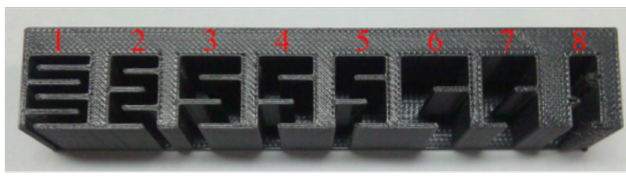

d)

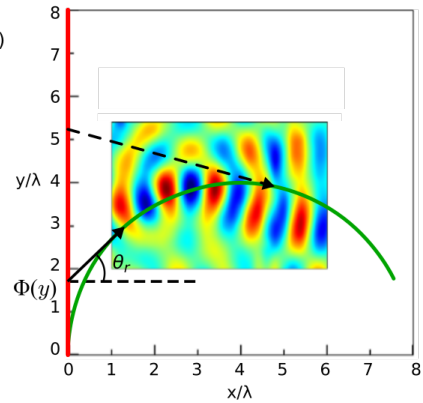

Figure 7. Shaping the reflection field, from $[22,23]$. The unit cell is easily parametrized (a) to obtain a phase shift profile ( $\mathbf{b}$, colors indicate the phase of the reflected field when a plane wave is incoming) combining different cells in an array (c). An arbitrary shape can be obtained, imposing different phase shift profiles, $\phi(y)$, to the reflected field (d). 
When considering an incident wave normal to the metasurface, the propagation angle of the reflected wave can be evaluated as $\theta_{r}=\arcsin \left(\frac{\lambda}{2 \pi} \frac{d \phi(y)}{d y}\right)$, where $\lambda$ is the wavelength of the incoming noise disturbance and $\phi$ is the acoustic phase shift. If the actual interest is not to achieve an exact reflection angle, but, as in a virtually scarfed nacelle application, an approximate value within a range, then the metasurface can be considered to be effectively broadband, and for multiples of the working frequency, it would still have the expected behavior. For example, when the desired behavior of the metasurface is to act like an inclined wall in the deflection of sound, the metasurface should be effective over a wide frequency range, provided that each frequency has a different reflection angle or a different virtual inclination of the wall.

While this concept has proven to be effective in a purely acoustic domain, deeper investigation is needed when the hosting fluid is moving, i.e., a background aerodynamic flow is present. In the virtually scarfed nacelle example, the metasurface would be grazed by the flow, and likely, a new "corrected" phase shift profile should be defined, taking into account, among other factors, the flow Mach number.

Near-total reflecting metasurfaces can also be exploited to achieve high transmission losses for cabin noise reduction. Although take-off and landing are the loudest moments, cruise is clearly the longest section of a civil aviation aircraft mission, and hence, the most interesting for passenger comfort improvements by noise reduction treatments. In this flight condition, excluding internal sources like air-conditioning systems, the main external cabin noise contributions are from the engines and the turbulent boundary layer excitations that produce vibrations that transmit to the fuselage stringers and skins that radiate noise inside the cabin.

With the increasing emphasis on noxious emission reduction and fuel consumption efficiency, there has been a renewed interest in different engine concepts beyond turbofans, like propfans, i.e., open rotor engines, to obtain the speed and performance of a turbofan with the intended fuel economy of a turboprop (for instance, the Airbus has patented aircraft designs with twin rear-mounted, contra-rotating propfans [53] and Boeing does as well [54]). One of the main outcomes that has so far discouraged the usage of propfans is the excessive cabin noise produced by this type of engine compared to the classic turbofan. The spectrum of noise measured inside the cabin would show discrete intense peaks at low-frequencies, i.e., in the $50-1500 \mathrm{~Hz}$ range, correlated with the engine rotators being at their fundamental and higher harmonics.

Most kinds of treatment for cabin noise reduction are difficult to implement due to the small space available in the cabin sidewalls and the strict limits on the overall aircraft weight. Several works have employed resonant membranes as an effective way of suppressing the low frequency noise inside the cabin. Metasurfaces composed by membranes with added masses [55] can be attached to aluminum panels to guarantee a huge effective dynamic mass at resonance frequency, suppressing the bending vibrations of the structure and inducing radiation cancellation from the surface due to the anti-phase motions at anti-resonance working frequencies. Furthermore, a double wall design involving resonant-decorated membranes can be adopted to improve the noise shielding efficiency at low frequencies, keeping the overall structure lightweight [56-59]. Lightweight structures, showing up to $50 \mathrm{~dB}$ in transmission loss at low frequencies that may also exhibit good mechanical properties have also been presented [60-62], in which a honeycomb frame is used as a core, covered by very thin rubber membranes. Multilayer sandwiches have shown very good acoustic insulation over the $50-1500 \mathrm{~Hz}$ range. This kind of layout seems to be the most promising due to its ability to yield the desired acoustic properties with a typically strong structure like a honeycomb without the need to add mass onto the membranes, hence respecting the prerequisite of lightness and not negatively affecting the aircraft's efficiency or maximum payload.

\subsection{Noise Trapping}

Noise trapping is one of the most intriguing metamaterial applications. It involves allowing a sound wave to enter into a domain where it is contained and forbidden to exit, creating a sort of 
insulation of the exterior domain from the trapped sound. The most interesting application, in the authors' opinion, of sound trapping [63] uses a one-way metasurface, made by a layer of Helmholtz resonators with different depths attached to a near-zero index space-coiling based metamaterial (in a zero index acoustic metamaterial, the sound wave is infinitely stretched in space, and it travels with extremely high phase velocity). The functioning principle is depicted in Figure 8. A normal incident wave with a uniform phase profile is allowed to pass through the zero index metamaterial. The phase array steers the wave phase profile, and once the wave is reflected it is no longer allowed to pass through the zero index layer due to its high incident angle selectivity.

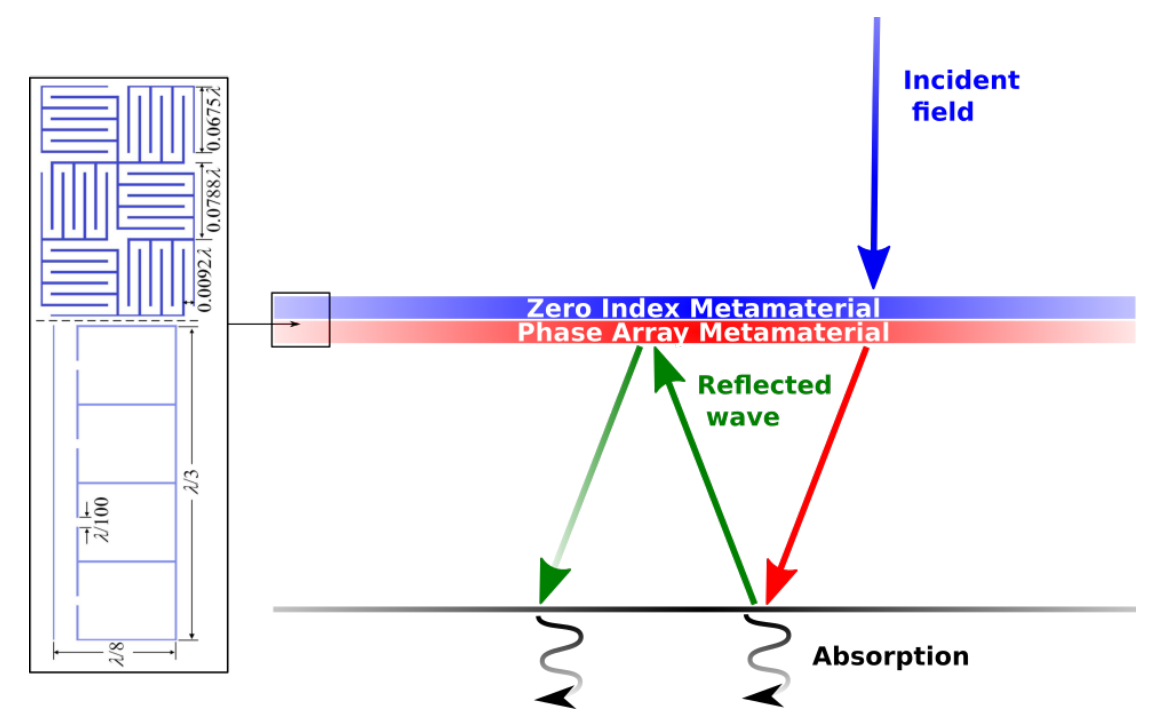

Figure 8. Noise trapping unit cell, from [63] and the functioning scheme of a noise trapping concept. The incident wave is allowed to pass through the metamaterial, and it is steered by the phase array into an absorbing material. The reflected fraction of the sound cannot escape and multiple reflections and absorption occur.

The described effect could very well turn out to be useful in aeronautical applications. It could be, for example, exploited as a way to enhance absorption and to be used in synergy with focusing devices or negatively refractive surfaces and absorbers. In a box delimited on one side by a suitable absorbing layer and on the other side by this metasurface, oriented such that the layer of resonator points is inside the considered domain, a sound wave can enter the domain through the zero index material, experiencing a redirection due to the phase shift obtained by tuning the depths of the resonators. Then, the wave can be partially absorbed by the absorbing layer. The reflected wave, however, is not allowed to exit the box due to the presence of the one-way metasurface. On the contrary, it is reflected back towards the absorber, enhancing its effect. The effectiveness of a non-perfect absorber with a non-negligible reflection coefficient can be improved, forcing the acoustic noise perturbation to repeatedly impinge on the absorbing device.

Nowadays, acoustic liners are used to reduce engine noise. Liners are applied on the internal walls of the engine nacelle, both in the intake and bypass ducts and use the Helmholtz resonance principle for the dissipation of incident acoustic energy. Placing a one-way metasurface at the interface between a liner and the internal side of the nacelle can result in an enhanced effect of the liner itself, especially at low frequencies, provided that sound waves cannot escape the domain occupied by the absorber. Obviously, one could imagine that to enhance the overall effect by both optimizing the metasurface and the liner positioning, and a totally new generation of liners needs to be designed, using, for example, a metamaterial absorber such as those described in the previous Section 2.1. The phase shift can also be tuned to maximize the number of possible reflections for a wide range of incident angles in the primary acoustic field. Note also that the phenomenon of the near-zero index is 
frequency-dependent, which means that the asymmetric acoustic behavior can be realized within a relatively narrow band, allowing for a shaping of the noise signature from the aircraft, as mentioned in Section 2.1, as multilayered structures can easily extend the working range of the concept.

A limitation of the design presented in [63] may be the resulting thickness, which is near to one half of the wavelength of interest, which would lead to the use of excessive thicknesses to be effective at the typical BPF (Blade Pass Frequency) of current turbofan engines and would thus not fit in the nacelle, especially if multiple layers need to be stacked. However, in the authors' opinion, this size could be further reduced by combining other metamaterial concepts. As the thickest part of the design is the phase-controlling metasurface, it could be replaced, e.g., by following $[22,23,38]$ or other similar concepts cited. The proposed scheme of designing a one-way metasurface would just need the coupling of a phase array and a non-dispersive, zero-index metamaterial, regardless of the particular implementation.

\subsection{Scattering Abatement (Cloaking)}

The term (acoustic) cloaking is commonly used to describe the ability of a device, the "cloak", to "hide" an obstacle from the incident noise perturbation field, thus preventing scattering from objects that may be embedded in such a domain.

The first examples of cloaking were published for electromagnetic waves in 2006 by Pendry [64] and Leonhardt [65]. Successively, the exploitation of the background idea of coordinate-transformation invariance of the governing equations has been transported to the acoustic domain by Cummer and Schurig [66]. This idea implies that any coordinate transformation-based deformation of the space can be physically created in real space with appropriate and typically complex distributions of acoustic properties of the propagation medium.

Norris $[67,68]$ gave a fundamental contribution to the theoretical development of the acoustic cloaking theory, showing that the first path discovered to obtain this effect was called "inertial cloaking" because it required a material to exhibit anisotropic density and led to an infinitely massive device. This a subcase of a more general theory-anisotropy can be distributed between the density and bulk modulus, the extremes defining inertial and pentamode metafluids, which both possess fluid-like properties that are not found in simple fluids. The first is defined by a tensorial anisotropic mass density and a scalar bulk modulus. The latter represents a solid structure, ideally behaving like a fluid, possessing five compliant, soft modes of deformation (connected to vanishing shear stiffness) and only one rigid mode (linked to the only non-null eigenvalue of the elasticity tensor representing compressibility). Pentamode metafluids may be engineered with a lattice of carefully interconnected solid struts, creating a structure that exhibits a quasi-vanishing shear stiffness [69,70] (Figure 9).

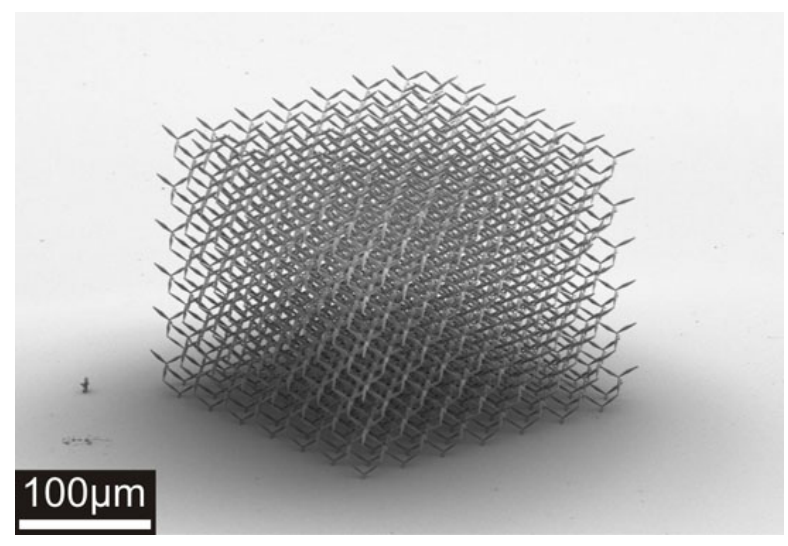

Figure 9. A pentamode material as realized by Kadic [69] with 3D laser lithography. The unit elements are linked to each other with connections that are $0.55 \mu \mathrm{m}$ thick. 
During the last decade, the scientific community produced a large number of papers on the topic, with numerical and theoretical papers and practical realizations of acoustic cloaking [71-78]. Alternative approaches exploiting coordinate transformations have been explored, like the so-called carpet cloaking [79-87], i.e., making an arbitrary-shaped surface act like a flat wall.

The transformation of the acoustic cloaking theory is valid only for quiescent media, hence when a moving fluid is present, the convective terms cause the governing equation to no longer be formally invariant under a coordinate transformation. Recent studies have tried to extend the existing theory of acoustic cloaking to the aeroacoustic domain, i.e., in the presence of flow [88-92] with interesting results (an example is given in Figure 10), even addressing complex turbulent flows via numerical optimization of the cloak design [93] and also exploiting a reinterpretation of the acoustics governing equations in four-dimensional space-time that exposes the relativistic structure of the phenomenon [94-98].

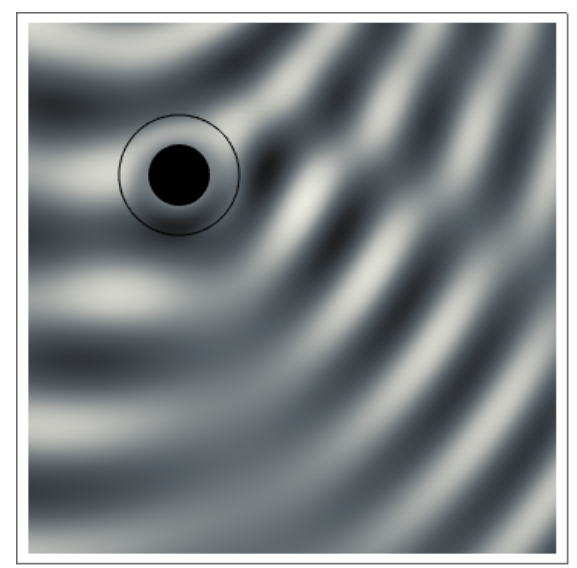

(a)

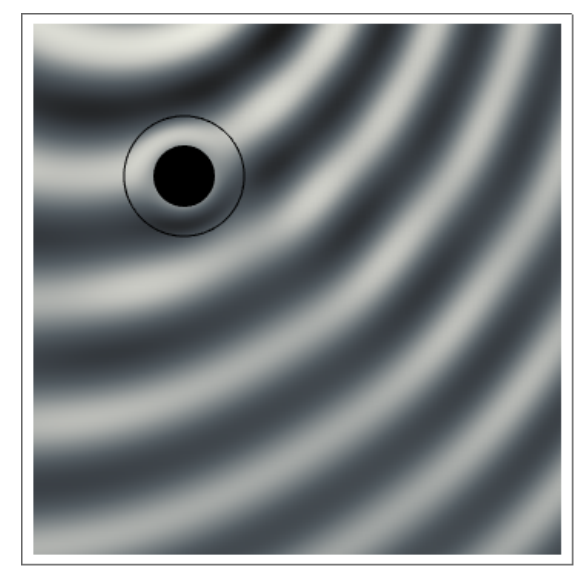

(b)

Figure 10. Acoustic cloaking in the presence of flow. The classic transformation acoustic theory defines a metamaterial that fails during abate scattering for the Mach number $M \neq 0$ (a). A correction of metamaterial parameters, as derived in [92], can recover the cloaking effect, even for $M=0.3$ (flow incoming from the right) (b).

So far, however, practical realizations have been limited to static acoustics and, for the most part, intended for carpet cloaking of small objects $[83,87]$ or based on large and/or massive devices $[99,100]$. Possibly the most interesting work has come from Mendez [78], who designed a feasible pentamode material for acoustic cloaking by topology optimization, starting from the desired metafluid acoustic characteristics, and numerically tested it.

Although the technology is not yet ready, one can think about the potential applications of cloaking an aircraft to reduce community noise. For instance, a virtually scarfed nacelle device could take advantage of the adoption of cloaking treatment of the upper side of the nacelle. It is easy to imagine a synergy in reflecting the acoustic energy towards the sky and avoiding further reflection from the upper front part of the nacelle, from the fuselage or from the wing. For this kind of application, cloaking devices should be able to effectively work in the presence of a mean, even turbulent, flow, their usage must not cause an excessive weight penalty, and their thickness must be smaller than the thickness of the nacelle and not negatively interfere with the overall aerodynamic behaviour.

Examples of recent theoretical work that may contribute to the evolution of transformation acoustics are the ones by Sklan, e.g., [101,102], which are among other topics focused on non-linear transformations. In these works, a unified theory of non-linear transformation metamaterials for optics is presented, covering all the combinations arising from considering a non-linear background medium 
and/or a non-linear transformation (that gives rise to non-linear metamaterials), and a brief extension to the acoustic field is also given.

\section{Challenges}

During the last decade, great effort has been made by the research community on the development of acoustic metamaterials and the ability to shape the acoustic field.

However, most of the so far presented works, even the concepts selected as the most promising for aeronautical applications, have dealt with quiescent hosting media, and have often focused on the development of noise-reducing material and structures for building acoustics. On the, still, long road towards the application of effective metamaterial concepts in the aeronautical framework for noise reduction, the first issue to be tackled is the inclusion of flows in models and simulations. As a moving fluid fundamentally affect acoustic wave propagation, corrections and modifications in models and concepts that depends on Mach numbers and/or flow field shapes, are expected to make them effective in the aeroacoustic domain.

Another challenge to face is the development of suitable numerical methods to simulate the behavior of acoustic metamaterials under aeronautical operating conditions. When it comes to evaluating the system response, one may consider analytical (or semi-analytical) approaches or numerical simulations. The former typically guarantees very fast evaluations, at the cost of a preliminary analytical resolution of the problem, which are not always available, especially for complex geometries and conditions with non-uniform complex background flows. Numerical approaches are more versatile, but versatility comes with a cost as well. Simulating acoustic metamaterials is a non-trivial challenge from the numerical point of view. Many concepts have labyrinthine complex micro-structures with audacious designs, and often, their behaviors rely on acousto-elastic interactions in structures where thermo-viscous losses are not negligible, thus they deeply affect the solution . A direct numerical simulation of such concepts, e.g., using the Finite Element Method (FEM) on fully detailed geometries, would result in computationally-expensive, high resource-demanding calculations, due to the fine mesh needed toaccurately reproduce the smallest geometrical details and solve the frequency of interest. This often does not allow for performance optimization testing, in which simulations are repeated a large number of times.

Hence, new efficient and accurate numerical models are needed that can combine advantages from different existing methods. This is the case, for example, of the one proposed in [103] for meta poro-elastic laminates with thin coatings, that couples the FEM, which simulates the response in the poro-elastic medium with inclusions, and the Bloch expansion, which simulates the surrounding media using a technique similar to the transfer matrix method to account for the coatings. Multiscale and homogenization modeling techniques are also attractive ways of addressing the issue when different scales are involved, such as microporous materials where one is interested in the macroscopic effect due to the microstructure of the system [104], and they can be adopted in a metamaterial design process $[105,106]$.

As a final remark, the actual manufacturing of metamaterial devices poses significant challenges. Often, metamaterials come with complex geometries that are needed to exploit particular physical phenomena, but may increase production complexity and hence, costs. It is obviously of fundamental importance to control the latter aspects to enable the deployment of metamaterial technologies in an industrial context. During the last two decades, additive manufacturing (AM) techniques have experienced a boost in terms of versatility and reliability, and several new processes have been developed that use a large variety of materials, including polymers and metals. The aerospace industry is introducing $\mathrm{AM}$, and through this technique, exploiting the possibilities offered to obtain lighter and still durable components with less geometrical restrictions in the design of the parts. In the authors' opinion, AM may be the key to successfully addressing the challenge of a low-cost manufacturing process that can handle the complex geometries required for metamaterial designs. 


\section{Conclusions}

A review of metamaterial concepts focusing on future aeronautical applications has been presented. The aeronautical community is concentrating its research efforts on the reduction of community noise produced by aircraft, and the significant potential shown in acoustics by metamaterials can undoubtedly give a contribution in this sense. Despite the huge body of literature on acoustic metamaterials, this potential still remain undisclosed for applications that involve a moving fluid. Starting from these considerations, and bearing in mind the restrictions on weight and size in the specific application field, concepts of acoustic metamaterials have been selected as the most promising for aircraft community noise reduction. The achievable results promised by metamaterials include, e.g., an enhanced and/or selective frequency noise absorption, even at low-frequencies, with subwavelength thickness, that can be exploited for a new generation of acoustic liners. Anomalous reflection from the nacelle's internal walls can create virtual scarfing effects from the intake of engines may also be adopted for cabin noise mitigation. Metamaterials will, ultimately, be able to unlock new degrees of freedom for designers, allowing them to shape the acoustic signature of aircrafts to make them less annoying for the community. To achieve effective implementation of the analyzed metadevices, deeper investigations on the effect of the presence of a background flow must be conducted, but also, the effects of metamaterial designs on the flow have to be studied. In fact, another detriment for effective applicability of a metamaterial concept in aeronautics will be, alongside excessive weight or size, a negative impact on aerodynamic efficiency; this must be carefully avoided. Since metamaterials derive their properties primarily from their structures, complex designs are often adopted and complex visco-thermal losses and acousto-elastic interactions are involved in the generation of the related metabehaviors. Numerically reproducing such phenomena can be hard, resulting in very expensive simulations if standard direct methods, such as FEM, are applied. It is clear that new, fast, and reliable numerical tools are needed to tackle the design and optimization processes of metamaterial devices that are necessary to produce the highest TRL of this new breakthrough technology.

Author Contributions: G.P., H.M. and L.B. performed the literature survey and collected the relevant references for the review. The original draft was prepared by G.P. and H.M., who also followed the review \& editing process together with P.G. and U.I. who also supervised the work as principal investigators of the Project AERIALIST.

Acknowledgments: This work was supported by the European Commission through the Project AERIALIST (AdvancEd aicRaft-noIse-AlLeviation devIceS using meTamaterials), Grant Agreement No. 723367.

Conflicts of Interest: The authors declare no conflict of interest.

\section{References}

1. Weiglhofer, W.S.; Lakhtakia, A. Introduction to Complex Mediums for Optics and Electromagnetics; SPIE Publications: Bellingham, WA, USA, 1999.

2. Cui, T.J.; Smith, D.; Liu, R. Metamaterials; Springer: Berlin/Heidelberg, Germany, 2010.

3. Veselago, V.G. The electrodynamics of substances with simultaneously negative values of $\epsilon$ and $\mu$. Sov. Phys. Uspekhi 1968, 10, 509. [CrossRef]

4. Pendry, J.B. Negative refraction makes a perfect lens. Phys. Rev. Lett. 2000, 85, 3966. [CrossRef] [PubMed]

5. Liu, Z.; Zhang, X.; Mao, Y.; Zhu, Y.Y.; Yang, Z.; Chan, C.T.; Sheng, P. Locally Resonant Sonic Materials. Science 2000, 289, 1734-1736. [CrossRef] [PubMed]

6. Li, J.; Chan, C.T. Double-negative acoustic metamaterial. Phys. Rev. E 2004, 70, 055602. [CrossRef] [PubMed]

7. Ding, Y.; Liu, Z.; Qiu, C.; Shi, J. Metamaterial with Simultaneously Negative Bulk Modulus and Mass Density. Phys. Rev. Lett. 2007, 99, 093904. [CrossRef] [PubMed]

8. Brunet, T.; Merlin, A.; Mascaro, B.; Zimny, K.; Leng, J.; Poncelet, O.; Aristégui, C.; Mondain-Monval, O. Soft 3D acoustic metamaterial with negative index. Nat. Mater. 2014, 14, 384. [CrossRef] [PubMed]

9. Lee, S.H.; Wright, O.B. Origin of negative density and modulus in acoustic metamaterials. Phys. Rev. B 2016, 93, 024302. [CrossRef] 
10. Ma, G.; Sheng, P. Acoustic metamaterials: From local resonances to broad horizons. Sci. Adv. 2016, 2, e1501595. [CrossRef] [PubMed]

11. Zhang, S.; Yin, L.; Fang, N. Focusing Ultrasound with an Acoustic Metamaterial Network. Phys. Rev. Lett. 2009, 102, 194301. [CrossRef] [PubMed]

12. Yang, Z.; Mei, J.; Yang, M.; Chan, N.H.; Sheng, P. Membrane-Type Acoustic Metamaterial with Negative Dynamic Mass. Phys. Rev. Lett. 2008, 101, 204301. [CrossRef] [PubMed]

13. Yang, Z.; Dai, H.M.; Chan, N.H.; Ma, G.C.; Sheng, P. Acoustic metamaterial panels for sound attenuation in the 50-1000 Hz regime. Appl. Phys. Lett. 2010, 96, 041906. [CrossRef]

14. Langfeldt, F.; Riecken, J.; Gleine, W.; Von Estorff, O. A membrane-type acoustic metamaterial with adjustable acoustic properties. J. Sound Vib. 2016, 373, 1-18. [CrossRef]

15. Mei, J.; Ma, G.; Yang, M.; Yang, Z.; Wen, W.; Sheng, P. Dark acoustic metamaterials as super absorbers for low-frequency sound. Nat. Commun. 2012, 3, 756. [CrossRef] [PubMed]

16. Cselyuszka, N.; Sečujski, M.; Crnojević-Bengin, V. Novel negative mass density resonant metamaterial unit cell. Phys. Lett. A 2015, 379, 33-36. [CrossRef]

17. Sheng, P. Coupled membranes with doubly negative mass density and bulk modulus. J. Acoust. Soc. Am. 2013, 134, 4026-4026. [CrossRef]

18. Fang, N.; Xi, D.; Xu, J.; Ambati, M.; Srituravanich, W.; Sun, C.; Zhang, X. Ultrasonic metamaterials with negative modulus. Nat. Mater. 2006, 5, 452-456. [CrossRef] [PubMed]

19. Liu, X.N.; Hu, G.K.; Huang, G.L.; Sun, C.T. An elastic metamaterial with simultaneously negative mass density and bulk modulus. Appl. Phys. Lett. 2011, 98, 251907. [CrossRef]

20. Graciá-Salgado, R.; García-Chocano, V.M.; Torrent, D.; Sánchez-Dehesa, J. Negative mass density and $\rho$-near-zero quasi-two-dimensional metamaterials: Design and applications. Phys. Rev. B 2013, 88, 224305. [CrossRef]

21. Lee, S.H.; Park, C.M.; Seo, Y.M.; Wang, Z.G.; Kim, C.K. Composite Acoustic Medium with Simultaneously Negative Density and Modulus. Phys. Rev. Lett. 2010, 104, 054301. [CrossRef] [PubMed]

22. Li, Y.; Liang, B.; Gu, Z.M.; Zou, X.Y.; Cheng, J.C. Reflected wavefront manipulation based on ultrathin planar acoustic metasurfaces. Sci. Rep. 2013, 3, 2546. [CrossRef] [PubMed]

23. Li, Y.; Jiang, X.; Li, R.Q.; Liang, B.; Zou, X.Y.; Yin, L.L.; Cheng, J.C. Experimental Realization of Full Control of Reflected Waves with Subwavelength Acoustic Metasurfaces. Phys. Rev. Appl. 2014, 2, 064002. [CrossRef]

24. Zhu, Y.F.; Fan, X.D.; Liang, B.; Yang, J.; Yang, J.; Yin, L.l.; Cheng, J.C. Multi-frequency acoustic metasurface for extraordinary reflection and sound focusing. AIP Adv. 2016, 6, 121702. [CrossRef]

25. Gu, Z.M.; Liang, B.; Zou, X.Y.; Cheng, J.C. Broadband diffuse reflections of sound by metasurface with random phase response. EPL (Europhys. Lett.) 2015, 111, 64003. [CrossRef]

26. Zhu, Y.; Fan, X.; Liang, B.; Cheng, J.; Jing, Y. Ultrathin Acoustic Metasurface-Based Schroeder Diffuser. Phys. Rev. X 2017, 7, 021034. [CrossRef]

27. Wang, W.; Xie, Y.; Konneker, A.; Popa, B.I.; Cummer, S.A. Design and demonstration of broadband thin planar diffractive acoustic lenses. Appl. Phys. Lett. 2014, 105, 101904. [CrossRef]

28. Xie, Y.; Wang, W.; Chen, H.; Konneker, A.; Popa, B.I.; Cummer, S.A. Wavefront modulation and subwavelength diffractive acoustics with an acoustic metasurface. Nat. Commun. 2014, 5, 5553. [CrossRef] [PubMed]

29. Liang, Z.; Feng, T.; Lok, S.; Liu, F.; Ng, K.B.; Chan, C.H.; Wang, J.; Han, S.; Lee, S.; Li, J. Space-coiling metamaterials with double negativity and conical dispersion. Sci. Rep. 2013, 3, 1614. [CrossRef] [PubMed]

30. Tang, K.; Qiu, C.; Ke, M.; Lu, J.; Ye, Y.; Liu, Z. Anomalous refraction of airborne sound through ultrathin metasurfaces. Sci. Rep. 2014, 4, 6517. [CrossRef] [PubMed]

31. Zhai, S.; Chen, H.; Ding, C.; Shen, F.; Luo, C.; Zhao, X. Manipulation of transmitted wave front using ultrathin planar acoustic metasurfaces. Appl. Phys. A 2015, 120, 1283-1289. [CrossRef]

32. Christensen, J.; Martín-Moreno, L.; García-Vidal, F.J. All-angle blockage of sound by an acoustic double-fishnet metamaterial. Appl. Phys. Lett. 2010, 97, 134106. [CrossRef]

33. Zhao, J.; Li, B.; Chen, Z.N.; Qiu, C.W. Redirection of sound waves using acoustic metasurface. Appl. Phys. Lett. 2013, 103, 151604. [CrossRef]

34. Zhao, S.D.; Wang, Y.S.; Zhang, C. A continuously tunable acoustic metasurface for transmitted wavefront manipulation. arXiv 2017, arXiv:1711.09701. [PubMed] 
35. Díaz-Rubio, A.; Tretyakov, S.A. Acoustic metasurfaces for scattering-free anomalous reflection and refraction. Phys. Rev. B 2017, 96, 125409. [CrossRef]

36. Leroy, V.; Strybulevych, A.; Lanoy, M.; Lemoult, F.; Tourin, A.; Page, J.H. Superabsorption of acoustic waves with bubble metascreens. Phys. Rev. B 2015, 91, 020301. [CrossRef]

37. Starkey, T.A.; Smith, J.D.; Hibbins, A.P.; Sambles, J.R.; Rance, H.J. Thin structured rigid body for acoustic absorption. Appl. Phys. Lett. 2017, 110, 041902. [CrossRef]

38. Cai, X.; Guo, Q.; Hu, G.; Yang, J. Ultrathin low-frequency sound absorbing panels based on coplanar spiral tubes or coplanar Helmholtz resonators. Appl. Phys. Lett. 2014, 105, 121901. [CrossRef]

39. Molerón, M.; Serra-Garcia, M.; Daraio, C. Visco-thermal effects in acoustic metamaterials: From total transmission to total reflection and high absorption. New J. Phys. 2016, 18, 033003. [CrossRef]

40. Lambert, J.; Champelovier, P.; Blanchet, R.; Lavandier, C.; Terroir, J.; Márki, F.; Griefahn, B.; Iemma, U.; Janssens, K.; Bisping, R. Human response to simulated airport noise scenarios in home-like environments. Appl. Acoust. 2015, 90, 116-125. [CrossRef]

41. NASA Glenn Research Center. Available online at https://www.nasa.gov/centers/glenn/images/content/ 83522main_fs003_fig3.gif (accessed on 22 May 2018).

42. Beck, B.S. Grazing incidence modeling of a metamaterial-inspired dual-resonance acoustic liner. Proc. SPIE 2014, 9064, 906421.

43. Beck, B.S.; Schiller, N.H.; Jones, M.G. Impedance assessment of a dual-resonance acoustic liner. Appl. Acoust. 2015, 93, 15-22. [CrossRef]

44. Mitchell, K.R.; Fuller, C.R. Design Optimization of Broadband Acoustic Liners through Finite Element Efficacy Studies. In Proceedings of the 21st AIAA/CEAS Aeroacoustics Conference, Dallas, TX, USA, 22-26 June 2015; American Institute of Aeronautics and Astronautics: Reston, VA, USA, 2015.

45. Li, Y.; Assouar, B.M. Acoustic metasurface-based perfect absorber with deep subwavelength thickness. Appl. Phys. Lett. 2016, 108, 063502. [CrossRef]

46. Chen, C.; Du, Z.; Hu, G.; Yang, J. A low-frequency sound absorbing material with subwavelength thickness. Appl. Phys. Lett. 2017, 110, 221903. [CrossRef]

47. Yang, M.; Chen, S.; Fu, C.; Sheng, P. Optimal sound-absorbing structures. Mater. Horiz. 2017, 4, 673-680. [CrossRef]

48. Tang, Y.; Ren, S.; Meng, H.; Xin, F.; Huang, L.; Chen, T.; Zhang, C.; Lu, T.J. Hybrid acoustic metamaterial as super absorber for broadband low-frequency sound. Sci. Rep. 2017, 7, 43340. [CrossRef] [PubMed]

49. Diez, M.; Iemma, U.; Marchese, V. A Sound-Matching-Based Approach for Aircraft Noise Annoyance Alleviation Via MDO. In Proceedings of the 13th AIAA/CEAS Aeroacoustics Conference (28th AIAA Aeroacoustics Conference), Rome, Italy, 21-23 May 2007.

50. Iemma, U.; Diez, M.; Leotardi, C.; Centracchio, F. On the use of noise annoyance as a design optimization constraint: the COSMA experience. In Proceedings of the 18th International Congress on Sound and Vibration (ICSV), Rio de Janeiro, Brazil, 10-14 July 2011.

51. Diez, M.; Iemma, U. Multidisciplinary conceptual design optimization of aircraft using a sound-matchingbased objective function. Eng. Optim. 2012, 44, 591-612. [CrossRef]

52. Montetagaud, F.; Montoux, S. Negatively Scarfed Intake: Design and Acoustic Performances. In Proceedings of the 11th AIAA/CEAS Aeroacoustics Conference, Monterey, CA, USA, 23-25 May 2005; American Institute of Aeronautics and Astronautics: Reston, VA, USA, 2005.

53. Gall, P.E.; Cros, C. Aircraft Having a Reduced Environmental Impact. U.S. Patent 2008/0258005 A1, 2008.

54. Moore, M.D.; Boren, K.L.; Marques, E.C. Airplane Configuration. U.S. Patent 8,087,607 B2, 2012.

55. Wang, X.; Huang, Z.; Han, F.; Feng, Z. Reduction of aircraft engine noise by covering surface acoustic metamaterials on sidewalls. In Proceedings of the 24th International Congress on Sound and Vibration (ICSV), London, UK, 23-27 July 2017.

56. Naify, C.J.; Chang, C.M.; McKnight, G.; Nutt, S.R. Experimental and FEA evaluation of membrane-type locally resonant acoustic metamaterial arrays for aircraft interior sound insulation. In Proceedings of the 17th AIAA/CEAS Aeroacoustics Conference (32nd AIAA Aeroacoustics Conference), Portland, Oregon, 5-8 June 2011.

57. Marinova, P.; von Estorff, O.; Lippert, S. Low-frequency air- and structure-borne sound insulation challenges for a counterrotating open-rotor aircraft. In Proceedings of the 21th International Congress on Sound and Vibration (ICSV), Beijing, China, 6-10 July 2014. 
58. Langfeldt, F.; Marinova, P.; Gleine, W.; Lippert, S.; von Estorff, O. Noise-shield with membrane-type metamaterials for low-frequency sound insulation. Part I: Analytical investigation of the multi-layered assembly. In Proceedings of the 40th German Annual Conference on Acoustics DAGA, Oldenburg, Germany, 10-13 March 2014.

59. Marinova, P.; Langfeldt, F.; Gleine, W.; Lippert, S.; von Estorff, O. Noise-shield with membrane-type metamaterials for low-frequency sound insulation. Part II: Numerical investigation of the full-scale acoustic panel assembly. In Proceedings of the 40th German Annual Conference on Acoustics DAGA, Oldenburg, Germany, 10-13 March 2014.

60. Sui, N.; Yan, X.; Huang, T.Y.; Xu, J.; Yuan, F.G.; Jing, Y. A lightweight yet sound-proof honeycomb acoustic metamaterial. Appl. Phys. Lett. 2015, 106, 171905. [CrossRef]

61. Wang, X.; Zhao, H.; Luo, X.; Huang, Z. Membrane-constrained acoustic metamaterials for low frequency sound insulation. Appl. Phys. Lett. 2016, 108, 041905. [CrossRef]

62. Gao, N.; Hou, H. Low frequency acoustic properties of a honeycomb-silicone rubber acoustic metamaterial. Modern Phys. Lett. B 2017, 31, 1750118. [CrossRef]

63. Jiang, X.; Liang, B.; Zou, X.Y.; Yang, J.; Yin, L.L.; Yang, J.; Cheng, J.C. Acoustic one-way metasurfaces: Asymmetric Phase Modulation of Sound by Subwavelength Layer. Sci. Rep. 2016, 6, 28023. [CrossRef] [PubMed]

64. Pendry, J.B.; Schurig, D.; Smith, D. Controlling electromagnetic fields. Science 2006, 312, 1780-1782. [CrossRef] [PubMed]

65. Leonhardt, U. Optical conformal mapping. Science 2006, 312, 1777-1780. [CrossRef] [PubMed]

66. Cummer, S.A.; Schurig, D. One path to acoustic cloaking. New J. Phys. 2007, 9, 45. [CrossRef]

67. Norris, A. Acoustic cloaking theory. Proc. R. Soc. A Math. Phys. Eng. Sci. 2008, 464, 2411-2434. [CrossRef]

68. Norris, A. Acoustic metafluids. J. Acoust. Soc. Am. 2009, 125, 839-849. [CrossRef] [PubMed]

69. Kadic, M.; Bückmann, T.; Stenger, N.; Thiel, M.; Wegener, M. On the practicability of pentamode mechanical metamaterials. Appl. Phys. Lett. 2012, 100, 191901. [CrossRef]

70. Kadic, M.; Bückmann, T.; Schittny, R.; Gumbsch, P.; Wegener, M. Pentamode Metamaterials with Independently Tailored Bulk Modulus and Mass Density. Phys. Rev. Appl. 2014, 2, 054007. [CrossRef]

71. Cai, L.W.; Sánchez-Dehesa, J. Analysis of Cummer-Schurig acoustic cloaking. New J. Phys. 2007, 9, 450. [CrossRef]

72. Chen, H.; Chan, C.T. Acoustic cloaking in three dimensions using acoustic metamaterials. Appl. Phys. Lett. 2007, 91, 183518. [CrossRef]

73. Cheng, Y.; Yang, F.; Xu, J.Y.; Liu, X.J. A multilayer structured acoustic cloak with homogeneous isotropic materials. Appl. Phys. Lett. 2008, 92, 151913. [CrossRef]

74. Torrent, D.; Sánchez-Dehesa, J. Broadband acoustic cloaks based on the homogenization of layered materials. Wave Motion 2011, 48, 497-504. [CrossRef]

75. Iemma, U.; Burghignoli, L. An integral equation approach to acoustic cloaking. J. Sound Vib. 2012, 331, 4629-4643. [CrossRef]

76. Scandrett, C.L.; Boisvert, J.E.; Howarth, T.R. Acoustic cloaking using layered pentamode materials. J. Acoust. Soc. Am. 2010, 127, 2856-2864. [CrossRef] [PubMed]

77. Chen, Y.; Liu, X.; Hu, G. Latticed pentamode acoustic cloak. Sci. Rep. 2015, 5, 15745. [CrossRef] [PubMed]

78. Méndez, C.G.; Podestá, J.M.; Lloberas-Valls, O.; Toro, S.; Huespe, A.E.; Oliver, J. Computational material design for acoustic cloaking. Int. J. Numer. Methods Eng. 2017, 112, 1353-1380. [CrossRef]

79. Pendry, J.B.; Li, J. An acoustic metafluid: Realizing a broadband acoustic cloak. New J. Phys. 2008, 10, 115032. [CrossRef]

80. Popa, B.I.; Cummer, S.A. Homogeneous and compact acoustic ground cloaks. Phys. Rev. B 2011, 83, 224304. [CrossRef]

81. Popa, B.I.; Zigoneanu, L.; Cummer, S.A. Experimental acoustic ground cloak in air. Phys. Rev. Lett. 2011, 106, 253901. [CrossRef] [PubMed]

82. Zhang, X.L.; Ni, X.; Lu, M.H.; Chen, Y.F. A feasible approach to achieve acoustic carpet cloak in air. Phys. Lett. A 2012, 376, 493-496. [CrossRef]

83. Zigoneanu, L.; Popa, B.I.; Cummer, S.A. Three-dimensional broadband omnidirectional acoustic ground cloak. Nat. Mater. 2014, 4, 352-355. [CrossRef] [PubMed] 
84. Zhu, J.; Chen, T.; Liang, Q.; Wang, X.; Jiang, P. Design of a broadband ultra-large area acoustic cloak based on a fluid medium. J. Appl. Phys. 2014, 116, 164906. [CrossRef]

85. Yang, Y.; Wang, H.; Yu, F.; Xu, Z.; Chen, H. A metasurface carpet cloak for electromagnetic, acoustic and water waves. Sci. Rep. 2016, 6, 20219. [CrossRef] [PubMed]

86. Esfahlani, H.; Karkar, S.; Lissek, H.; Mosig, J.R. Acoustic carpet cloak based on an ultrathin metasurface. Phys. Rev. B 2016, 94, 014302. [CrossRef]

87. Faure, C.; Richoux, O.; Félix, S.; Pagneux, V. Experiments on metasurface carpet cloaking for audible acoustics. Appl. Phys. Lett. 2016, 108, 064103. [CrossRef]

88. Huang, X.; Zhong, S.; Stalnov, O. Analysis of scattering from an acoustic cloak in a moving fluid. Acoust. Soc. Am. 2014, 135, 2571-2580. [CrossRef] [PubMed]

89. Ryoo, H.; Jeon, W. Effect of compressibility and non-uniformity in flow on the scattering pattern of acoustic cloak. Sci. Rep. 2017, 7, 2125-2136. [CrossRef] [PubMed]

90. Iemma, U.; Carley, M.; Pellegrini, R. Tailoring Acoustic Metamaterials to Aeroacoustic Applications. In Proceedings of the 43th International Congress and Exposition on Noise Control Engineering (Inter-Noise), Melbourne, Australia, 16-19 November 2014.

91. Iemma, U. Theoretical and Numerical Modeling of Acoustic Metamaterials for Aeroacoustic Applications. Aerospace 2016, 3, 15. [CrossRef]

92. Iemma, U.; Palma, G. Aeroacoustic Design of Metafluid Devices. In Proceedings of the 24th International Congress on Sound and Vibration (ICSV), London, UK, 23-27 July 2017.

93. Huang, X.; Zhong, S.; Liu, X. Acoustic invisibility in turbulent fluids by optimised cloaking. J. Fluid Mech. 2014, 749, 460-477. [CrossRef]

94. García-Meca, C.; Carloni, S.; Barceló, C.; Jannes, G.; Sánchez-Dehesa, J.; Martínez, A. Analogue transformations in physics and their application to acoustics. Sci. Rep. 2013, 3, 2009. [CrossRef] [PubMed]

95. García-Meca, C.; Carloni, S.; Barceló, C.; Jannes, G.; Sánchez-Dehesa, J.; Martínez, A. Space-time transformation acoustics. Wave Motion 2014, 51, 785-797. [CrossRef]

96. García-Meca, C.; Carloni, S.; Barceló, C.; Jannes, G.; Sánchez-Dehesa, J.; Martínez, A. Analogue transformation acoustics and the compression of spacetime. Photonics Nanostruct. Fundam. Appl. 2014, 4, 312-318. [CrossRef]

97. Iemma, U.; Palma, G. Analogue Transformation Acoustics in Aeronautics. In Proceedings of the 46th International Congress and Exposition on Noise Control Engineering (Inter-Noise), Hong Kong, China, 27-30 August 2017.

98. Iemma, U.; Palma, G. On the Use of the Analogue Transformation Acoustics in Aeroacoustics. Math. Probl. Eng. 2017, 2017, 8981731. [CrossRef]

99. Zhang, S.; Xia, C.; Fang, N. Broadband Acoustic Cloak for Ultrasound Waves. Phys. Rev. Lett. 2011, 106, 024301. [CrossRef] [PubMed]

100. Yang, S.K.; Lin, J.C.; Cheng, J.W. The Experimental Realization of an Acoustic Cloak in Air with a Meta-Composite Shell. Appl. Sci. 2017, 7, 456. [CrossRef]

101. Sklan, S.R.; Li, B. A Unified Approach to Nonlinear Transformation Materials. Sci. Rep. 2018, 8, 4436-4448. [CrossRef] [PubMed]

102. Sklan, S.R. Splash, pop, sizzle: Information processing with phononic computing. AIP Adv. 2015, 5, 053302. [CrossRef]

103. Gaborit, M.; Schwan, L.; Dazel, O.; Groby, J.P.; Weisser, T.; Göransson, P. Coupling FEM, Bloch Waves and TMM in Meta Poroelastic Laminates. Acta Acust. United Acust. 2018, 104, 220-227. [CrossRef]

104. Gao, K. Multiscale Modelling of Acoustic Porous Materials. Ph.D. Thesis, Technische Universiteit Eindhoven, Eindhoven, The Netherlands, 2016.

105. Roca, D.; Lloberas-Valls, O.; Cante, J.; Oliver, J. A computational multiscale homogenization framework accounting for inertial effects: Application to acoustic metamaterials modelling. Comput. Methods Appl. Mech. Eng. 2018, 330, 415-446. [CrossRef]

106. Sridhar, A.; Kouznetsova, V.G.; Geers, M.G.D. Homogenization of locally resonant acoustic metamaterials towards an emergent enriched continuum. Comput. Mech. 2016, 57, 423-435. [CrossRef] [PubMed]

(C) 2018 by the authors. Licensee MDPI, Basel, Switzerland. This article is an open access article distributed under the terms and conditions of the Creative Commons Attribution (CC BY) license (http:/ / creativecommons.org/licenses/by/4.0/). 